\title{
Efeito do uso de leite fortificado com ferro e vitamina C sobre os níveis de hemoglobina e condição nutricional de crianças menores de 2 anos
}

\author{
Effects of vitamin $C$ and iron-fortified milk use on hemoglobin levels and \\ nutritional condition of children cared for in day
}

\author{
Marco A. A. Torres, Kazue Sato, Neil Ferreira Lobo, Suzana de Souza Queiroz \\ Núcleo de Nutrição do Centro de Referência de Saúde da Mulher. Nutrição. \\ Alimentação e Desenvolvimento Infantil da Secretaria de Estado da Saúde de São Paulo- \\ São Paulo-Brasil (M. A. A. T., K. S.), \\ Departamento de Medicina Preventiva da Universidae Federal de São Paulo - São Paulo - \\ Brasil (N.F.L.), \\ Departamento de Pediatria da Faculdade de Medicina de Botucatu - Botucatu - Brasil (S.S.Q.)
}

\begin{abstract}
Foi avaliado o impacto do uso do leite em pó integral fortificado com $9 \mathrm{mg}$ de ferro $\theta 65 \mathrm{mg}$ de vitamina $\mathrm{C}$ para cada 100 $g$ de po, sobre os niveis de hemoglobina de crianças menores de 2 anos, em 107 crianças de creches municipais e 228 de uma Unidade Básica de Sáude (UBS), por um período de 6 meses. Antes de se iniciar a intervençảo, $66,4 \%$ das crianças das creches $\theta 72,8 \%$ da UBS apresentavam níveis de hemoglobina inferiores a $11,0 \mathrm{~g} / \mathrm{dl}$. Ao final dos 6 meses de uso do leite fortificado, esses percentuais reduziram-se para $20,6 \%$ nas creches e $18,0 \%$ na UBS. A média da hemoglobina, antes de se iniciar o experimento, foi de $10,3 \mathrm{~g} / \mathrm{dl}$ nas creches e $10,5 \mathrm{~g} / \mathrm{dl}$ na UBS. Decorridos 6 meses esses valores subiram para $11,6 \mathrm{~g} / \mathrm{dl}$ nas duas populaçōes estudadas. Em relaçäo à condição nutricional, avaliada pelo critério de Gomez. verificou-se que, nas creches, $57 \%$ das crianças acompanhadas apresentaram melhoria na sua condiçāo nutricional, 41 , $1 \%$ ficaram inalteradas $\theta$ apenas $1,9 \%$ pioraram. Na UBS, $11,4 \%$ apresentaram melhora, $70,6 \%$ ficaram inalteradas e $18 \%$ pioraram, o que mostrou uma diferença de resposta quanto à recuperaçāo da condiçāo nutriçional, quando o leite enriquecido foi utilizado em ambiente aberto e fechado. Concluiu-se que a utilizaçāo de alimentos fortificados apresentase como excelente alternativa para o controle da carência de ferro em populaçбes de crianças menores de 2 anos.
\end{abstract}

Alimentos fortificados. Substitutos do leite humano. Anemia ferropriva, prevençâo \& controle. Hemoglobina, análise.

\section{Introdução}

A anemia é a patologia de maior prevalência em todo o mundo, principalmente na população infantil de países em desenvolvimento ${ }^{14}$, onde se registram freqüências quatro vezes maiores que as encontradas em países desenvolvidos ${ }^{5}$. No Brasil, na faixa etária de menores de 2 anos, a proporção de anêmicos situa-se entre $50 \%$ a $83,5 \%$ 2,8.11.20,23,24,26.

A carência de ferro é a principal responsável pelas elevadas prevalências de anemia encontradas $^{5}$. Em menores de 2 anos, mais que em qualquer outra idade, o principal determinante do status de ferro no organismo é nutricional; esse é um período de crescimento rápido, geralmente associado à bai$\mathrm{xa}$ ingestão desse elemento. Esta dependência do ferro proveniente da dieta é maior a partir dos 6 meses, quando os estoques do mineral no organismo estão depletados, mas pode aparecer mais precocemente, como nos prematuros e recém-nascidos de baixo peso para a idade gestacional, por causa das baixas reservas que essas crianças apresentam ao nascer ${ }^{12}$. Em Sāo Paulo, a prática do aleitamento materno, fonte de ferro de alta biodisponibilidade, que atualmente nāo atinge a média de 2,5 meses, segundo dados do Sistema de Vigilância Nutricional da Secretaria de Estado da Saúde de São Paulo ${ }^{18}$. É substituído pelo leite de vaca, sabidamente deficiente no oligo-elemento e causador de micro-hemorragias no trato gastrointestinal.

Na cidade de São Paulo foram encontradas dietas deficientes em ferro em $48 \%$ das crianças menores de

Separatas/Reprints: Marco Antonio de Almeida Torres - Rua: Dr. Augusto de Miranda, 1297 - 05026-001 - Sāo Paulo, SP - Brasil Ediçāo subvencionada pela FAPESP. Processo 95/2290-6.

Recebido em 17.2.1994. Reapresentado em 8.3.1995. Aprovado em 10.7.1995. 
5 anos, sendo esta situação ainda mais frequiente nos dois primeiros anos de vida ${ }^{11}$. Como consequência, $59,9 \%$ dos menores de 2 anos que freqüentam as Unidades Básicas de Saúde (UBS), em todo o Estado de São Paulo, apresentam níveis de hemoglobina inferiores a $11,0 \mathrm{~g} / \mathrm{dl} \mathrm{e} 25,1 \%$, inferiores a $9,5 \mathrm{~g} / \mathrm{dl}^{24}$.

Poucos são os estudos, no país, preocupados em testar ações que visem à redução dos níveis de anemia na populaçāo. A suplementação medicamentosa foi testada em São Paulo ${ }^{25}$ e Recife ${ }^{17} \mathrm{com}$ resultados semelhantes; a intervençâo mostrou-se eficaz em termos de elevação dos níveis da hemoglobina das crianças avaliadas. Dois fatores, entretanto, impediram a obtenção de melhores resultados: a baixa aderência da população à proposta de intervençăo e o "esquecimento" em oferecer diariamente ofármaco à criança.

A fortificação de alimentos é outra alternativa de intervenção, recomendada principalmente para localidades onde se encontram elevadas prevalências $^{4,10,12}$, comoé o caso do Brasil. Esta prática pode ser utilizada para toda a populaçăo, ou em grupos populacionais especificos, sem exigir, necessariamente, a cooperação do beneficiário. A experiência chilena com o leite em pó integral, enriquecido com ferro e vitamina $\mathrm{C}$, demonstrou a eficácia deste tipo de intervençāo ${ }^{13,22}$.

O objetivo do presente estudo foi avaliar a eficácia do uso do leite em po fortificado com ferro e vitamina $\mathrm{C}$, como proposta de intervenção no combate à anemia por deficiência de ferro, em crianças menores de 2 anos, matriculadas em creches municipais, comparativamente à crianças da mesma faixa etária, acompanhadas em (UBS). Além disso, a utilização do leite enriquecido foi relacionada com possíveis alteraçóes nos níveis de hemoglobina e na condição nutricional das crianças acompanhadas.

\section{Materlal e Método}

Foram acompanhadas longitudinalmente 335 crianças com idades compreendidas entre 6 e 23 meses, sendo 107 de 13 creches de 3 municípios da Grande Sāo Paulo (Caieiras, Francisco Morato e Mairiporã) e 228 captadas pela demanda espontânea da UBS do Município de Ibiúna, situado a $70 \mathrm{~km}$ de São Paulo.

Excluiu-se da amostra todos os lactentes e préescolares portadores de qualquer tipo de patologia que pudesse interferir no processo normal de crescimento e desenvolvimento.

Antes de se iniciar a intervençăo, as crianças foram submetidas a avaliações clínica e antropométrica e à coleta de sangue para dosagem da hemoglobina. A partir daí, as 335 crianças passaram a receber o leite em pó fortificado com $9 \mathrm{mg}$ de ferro e $65 \mathrm{mg}$ de vitamina $C$ por $100 \mathrm{~g}$ de po (depois de preparada, cada mamadeira de $250 \mathrm{ml}$ oferecia $3 \mathrm{mg}$ de ferro e $13,6 \mathrm{mg}$ de vitamina $\mathrm{C}$ ).

Nas creches foi feita a simples substituição do leite em pó integral, oferecido anteriormente, pelo fortificado. Não houve modificação nos cardápios, nem qualquer tipo de orientaçăo sobre o preparo e melhor aproveitamento dos alimentos.

A criança permanecia na creche das 7 às 17 horas, nos 5 dias úteis da semana e durante este período recebia entre 2 a 3 mamadeiras de $250 \mathrm{ml}$ de leite. Durante todo o experimento foram anotados, em fichas individuais, dados sobre a aceitaçăo e quantidade de leite ingerida diariamente, presença na creche e patologias apresentadas com o seu respectivo tempo de duraçăo.

Na UBS passou-se a distribuir para cada componente da amostra o leite fortificado nas seguintes quantidades: $4 \mathrm{~kg} / \mathrm{mês}$ para menores e $2 \mathrm{~kg} /$ mês aos maiores de um ano, que permitia a ingestāo de 3 a 4 mamadeiras de $250 \mathrm{ml} / \mathrm{dia}$. As mães foram orientadas quanto ao preparo das mamadeiras na concentração correta.

Todas as crianças foram submetidas a mais duas avaliações clínicas, nutricionais e hematológicas, 3 e 6 meses após o início da intervenção.

A dosagem da hemoglobina foi realizada pelo método da cianometa-hemoglobina ${ }^{7}$, com leitura em duplicata. Para caracterização da anemia foi adotado o critério da $\mathrm{OMS}^{14}$, que estabelece o limite de $11,0 \mathrm{~g} / \mathrm{dl}$ para as idades estudadas.

A condiçăo nutricional foi avaliada pelo critério de Gomez ${ }^{6}$, utilizando-se como padrão de referência as recomendaçōes da OMS ${ }^{15}$.

Na UBS, ao término da pesquisa, ainda houve disponibilidade de uma quantidade de leite suficiente para prolongar a suplementaçăo para um pequeno grupo de crianças. Para tanto, seleciou-se as 39 que apresentaram evoluções pouco satisfatórias durante os 6 meses iniciais e prolongou-se a intervenção por mais 3 meses, quando nova avaliação foi efetuada.

Para a análise estatística dos resultados foram realizados os seguintes testes:

1. Teste do Qui-Quadrado ${ }^{19}$, quando comparou-se os resultados de creches e UBS quanto ao percentual de crianças com anemia nos momentos inicial, 3 meses e 6 meses de estudo.

2. Teste de Goodman', para comparar os resultados de creches e UBS quanto às percentagens de crianças em cada um dos níveis de hemoglobina considerados.

3. Análise de variância para grupos năo independentes ${ }^{21}$, com a finalidade de comparar as taxas de hemoglobina nos momentos inicial, 3 meses e 6 meses de uso do leite fortificado. Esta análise, quando mostrou diferença significante, foi complementada pelo teste de Tukey ${ }^{21}$.

4. Teste $t$ de Student ${ }^{21}$ para as duas amostras independentes, com o objetivo de comparar as 
crianças das creches e da UBS, com relação aos valores das hemoglobinas. O mesmo teste, para amostras não independentes, foi aplicado para comparar, em separado para creches e UBS, as hemoglobinas nos momentos inicial e final do estudo.

5. Teste de Mann-Whitney ${ }^{19}$ para comparar creches e UBS, em relaçăo aos valores da diferença percentual $(\Delta \%)$ calculada para a hemoglobina, a partir dos momentos inicial e final, adotando-se o primeiro como referência. Estes valores foram calculados pela fórmula:

$$
\Delta \%=\frac{\text { valor final }- \text { valor inicial }}{\text { valor inicial }}=\times 100
$$

6. Teste de Cochram ${ }^{19}$, para comparar, na UBS, o percentual de crianças com anemia, nos momentos inicial, 3 meses, 6 meses e 9 meses de implantação do projeto.

7. Teste de concordância de Kappa ${ }^{9}$, com o objetivo de comparar, tanto nas creches como na UBS, as frequências de crianças, segundo sua condição nutricional, pelo critério de Gomez. Este teste foi complementado pelo teste de $\mathrm{McNemar}{ }^{16,}$ com a finalidade de estudar as frequências de crianças situadas acima ou abaixo da diagonal de concordância do teste de Kappa.

\section{Resultados}

Antes de se iniciar a intervenção, $70,7 \%$ de todas as crianças estudadas apresentavam dosagens de hemoglobina inferiores a $11,0 \mathrm{~g} / \mathrm{dl}$ (Tabela 1). Este percentual foi ligeiramente superior na UBS $(72,8 \%)$ que nas creches $(66,4 \%)$. O teste do Qui-Quadrado, calculado a partir dos valores absolutos, não mostrou diferença significante entre a ocorrência de anemia nas crianças das creches e da UBS.

Nas creches, a idade das crianças acompanhadas variou de 5 a 24 meses, com uma média de 13 meses. Na UBS, variou de 5 a 17 meses, com uma média de 8,5 meses.

A Tabela 2 mostra que o percentual de crianças com hemoglobinas inferiores a $9,5 \mathrm{~g} / \mathrm{dl}$ foi de $29,0 \%$

Tabela 1- Prevalências de anemia, anterior à intervençăo, em crianças menores de 2 anos de creches municipais da Grande São Paulo $\theta$ da Unidade Básica de Saúde (UBS) de Ibiúna, SP.

\begin{tabular}{lccc}
\hline \multirow{2}{*}{$\begin{array}{l}\text { Local do } \\
\text { Estudo }\end{array}$} & Amostra & \multicolumn{2}{c}{ Crianças com Anemia } \\
\cline { 3 - 4 } & $\mathrm{N}$ & $\mathrm{N}$ & $\%$ \\
\hline Creches & 107 & 71 & 66,4 \\
UBS & 228 & 166 & 72,8 \\
\hline Total & 335 & 237 & 70,7 \\
\hline
\end{tabular}

$\mathrm{N}$ - Número amostral

Teste do Qul-Quadrado $-X^{2}$ calc. $=1,46$ (NS) nas creches e de $8,8 \%$ na UBS. Por outro lado, na UBS, a grande maioria das crianças $(64,0 \%)$ apresentou níveis entre $9,5 \mathrm{e} 10,9 \mathrm{~g} / \mathrm{dl}$. No total, apenas $29,3 \%$ apresentaram hemoglobinas superiores aos $11,0 \mathrm{~g} / \mathrm{d} 1$. A aplicação do teste de Goodman apontou diferença estatisticamente significante na freqüência dos níveis de hemoglobina encontrados nas creches e UBS $(\mathrm{P}<0,001)$ e que esta diferença encontra se nas faixas inferiores a $9,5 \mathrm{~g} / \mathrm{dl}(\mathrm{P}<0,001)$ e entre 9,5 e $10,9 \mathrm{~g} / \mathrm{dl}(\mathrm{P}<0,01)$.

A Tabela 3 mostra a evolução das médias das hemoglobinas nos três exames realizados durante $o$ estudo. Entre os momentos inicial e final verificouse incremento médio de $1,3 \mathrm{~g} / \mathrm{dl}$ nas creches e de 1,1 $\mathrm{g} / \mathrm{dl}$ na UBS de Ibiúna, com um valor médio final de $11,6 \mathrm{~g} / \mathrm{dl}$ nos dois grupos acompanhados. A análise de variância para grupos não independentes, nos três momentos do estudo (inicial x 3 meses $\times 6$ meses) detectou diferença significante tanto nas creches $(\mathrm{P}<0,001)$ quanto na UBS $(\mathrm{P}<0,001)$, e foi complementada pelo teste de Tukey (inicial $<3$ meses $<6$ meses). Por outro lado, o teste " $t$ ", ao comparar as média das hemoglobinas nas creches e UBS, nos três momentos do estudo, não mostrou diferenças significantes entre elas.

A Tabela 4 mostra a evoluçăo dos valores médios das hemoglobinas, e seus respectivos desvios-padrāo, entre os momentos inicial e final da intervençāo, para os grupos de crianças com e sem anemia. Nas creches, o incremento foi de $1,9 \mathrm{~g} / \mathrm{dl}$ em crianças com anemia e de $0,4 \mathrm{~g} / \mathrm{dl}$, nas sem anemia, e na UBS, de $1,4 \mathrm{~g} / \mathrm{dl}$ nas crianças anêmicas e de $0,4 \mathrm{~g} /$ dl nas não anêmicas. A aplicação do teste " $\mathrm{t}$ " pareado, para comparar as médias das hemoglobinas nos momentos inicial e após 6 meses de intervençāo, mostrou diferença significante para o grupo de crianças anêmicas, tanto nas creches como na UBS e somente para as crianças não anêmicas na UBS

Tabela 2 - Distribuiçăo dos níveis de hemoglobina, anterior à intervençąo, em crianças menores de 2 anos, de creches municipais da Grande Săo Paulo e da Unidade Básica de Saúde (UBS) de Ibiúna, SP.

\begin{tabular}{lcccr}
\hline $\begin{array}{c}\text { Níveis de } \\
\text { Hemoglobina } \\
\text { ( } \mathrm{g} / \mathrm{dl})\end{array}$ & \multicolumn{2}{c}{ Creches } & \multicolumn{2}{c}{ UBS } \\
\cline { 2 - 5 } & $\mathrm{N}$ & $\%$ & $\mathrm{~N}$ & $\%$ \\
\hline$<9,5$ & 31 & 29,0 & 20 & 8,8 \\
$9,5 \geq 10,9$ & 40 & 37,4 & 146 & 64,0 \\
$\geq 11,0$ & 36 & 33,6 & 62 & 27,2 \\
\hline Total & 107 & 100,0 & 228 & 100,0 \\
\hline $\mathrm{N}-$ Número amostral & & & \\
Teste de Goodman & & & \\
$\mathrm{Y}^{2}$ calculado=27,51(P<0,001) & & \\
Contrastes entre locais & \\
$<9,5-\mathrm{G}$ calculado $=4,24$ (P<0,01) & \\
$9,5 \geq 10,9-G$ calculado $=4,71$ (P<0,01) \\
$\geq 11,0-\mathrm{G}$ calculado $=1,19$ (NS)
\end{tabular}


Tabela 3 - Evolução das médias das hemoglobinas (Hb), nos momentos inicial (0), após 3 (3) e 6 meses (6) de uso do leite fortificado, em crianças menores de 2 anos. Grande São Paulo e da Unidade Básica de Saúde (UBS) de lbiúna, SP.

\begin{tabular}{|c|c|c|c|c|}
\hline Local & Momento & $\begin{array}{c}\text { Amostra } \\
\mathrm{N} .\end{array}$ & $\frac{\mathrm{Hb}(\mathrm{g} / \mathrm{d})}{\mathrm{X} \pm \mathrm{DP}}$ & $\begin{array}{c}\text { Testes estatísticos } \\
(0 \times 3 \times 6)\end{array}$ \\
\hline Creches & $\begin{array}{l}0 \\
3 \\
6\end{array}$ & $\begin{array}{l}107 \\
107 \\
107\end{array}$ & $\begin{array}{l}10,3 \pm 1,42 \\
11,0 \pm 1,27 \\
11,6 \pm 1,13\end{array}$ & $\begin{array}{c}\text { Fcalc. }=88,40(P<0,001) \\
\text { Tukey }=0<3<6\end{array}$ \\
\hline UBS & $\begin{array}{l}0 \\
3 \\
6\end{array}$ & $\begin{array}{l}241 \\
241 \\
241\end{array}$ & $\begin{array}{l}10,5 \pm 0,90 \\
11,2 \pm 0,88 \\
11,6 \pm 0,87\end{array}$ & $\begin{array}{c}\text { Fcalc. }=169,64(P<0,001) \\
\text { Tukey }=0<3<6\}\end{array}$ \\
\hline \multicolumn{2}{|c|}{$\begin{array}{l}\text { N- Número amostral } \\
X \text { - Média } \\
\text { DP - Desvio-padrāo }\end{array}$} & \multicolumn{2}{|c|}{$\begin{array}{l}\text { Teste "t" ( creches x UBS) } \\
0-" \mathrm{t}=1,16 \text { (NS) } \\
3-" \mathrm{t}=1,40 \text { (NS) } \\
6-" \mathrm{t} "=0,21 \text { (NS) }\end{array}$} & \\
\hline
\end{tabular}

Tabela 4 - Evolução das médias das hemoglobinas, nos momentos inicial (I) e final (F) da intervenção, em crianças menores de 2 anos, com e sem anemia de creches municipais da Grande São Paulo e da Unidade Básica de Saúde (UBS) de lbiúna, SP.

\begin{tabular}{|c|c|c|c|c|c|}
\hline \multirow{3}{*}{$\begin{array}{l}\text { Local } \\
\text { do } \\
\text { Estudo }\end{array}$} & \multirow{3}{*}{$\begin{array}{c}\text { Condiçāo } \\
\text { Hematologgica }\end{array}$} & \multirow{3}{*}{$\begin{array}{c}\text { Amostra } \\
\text { N }\end{array}$} & \multicolumn{2}{|c|}{ Momento do Estudo } & \multirow{3}{*}{$\begin{array}{c}\text { Teste "t" pareado } \\
\text { (I X F) } \\
\text { "t" calculado }\end{array}$} \\
\hline & & & I & $F$ & \\
\hline & & & $X \pm D P$ & $X \pm D P$ & \\
\hline \multirow{2}{*}{ Creches } & Anêmicos & 71 & $9,5 \pm 1,06$ & $11,4 \pm 1,17$ & $14,86(P<0,001)$ \\
\hline & Não anêmicos & 36 & $11,7 \pm 0,73$ & $12,0 \pm 0,92$ & 1,93 (NS) \\
\hline \multirow{2}{*}{ UBS } & Anêmicos & 166 & $10,1 \pm 0,67$ & $11,5 \pm 0,89$ & $17,28(P<0,001)$ \\
\hline & Năo anêmicos & 62 & $11,5 \pm 0,51$ & $11,89 \pm 0,86$ & $3,76(P<0,001)$ \\
\hline
\end{tabular}

$\mathrm{N}$ - Número amostral

$X$ - Média

DP - Desvio Padräo

Teste " $\mathrm{t}$ " independente (creches $\times$ UBS)

Anêmicos

momento inicial $-t$ calculado $=4,36(P<0,001)$

após 6 meses - t calculado $=0,77$ (NS)

Teste de Mann-Whitney (creches $x$ UBS)

Anêmicos

$Z$ calculado $=3,82(P<0,001)$

CRECHE > UBS

Não anêmicos

momento inicial - $t$ calculado $=1,36$ (NS)

após 6 meses - $t$ calculado $=0,37$ (NS)

Năo anêmicos

$\mathrm{Z}$ calculado $=0,59$ (NS)

$(\mathrm{P}<0,001)$. Por outro lado, o teste " $\mathrm{t}$ " ao comparar, para creches e UBS, a média das hemoglobinas nos momentos inicial e após 6 meses de uso do leite fortificado, só detectou diferenças significantes $(\mathrm{P}<0,001)$ para crianças anêmicas no momento anterior à intervenção. O teste de Mann- Whitney, ao comparar os valores da diferença percentual $(\Delta \%)$, calculado para as hemoglobinas, entre os momentos inicial e final, adotando o primeiro como referência, encontrou incrementos significantes $(\mathrm{P}<0,001)$ para o grupo de anêmicos e este incremento foi superior nas crianças das creches.

A evolução do número e percentual de crianças com hemoglobinas inferiores a $11,0 \mathrm{~g} / \mathrm{dl}$ no período de utilizaçăo do leite fortificado, estăo representados na Figura 1. Os resuitados mostram comportamento semelhante nos dois estudos, com um acentuado decréscimo do percentual de crianças com anemia. Ao final da intervençāo constatou-se que, nas creches, $20,6 \%$ das crianças permaneciam anêmicas, enquanto que, na UBS, este percentual foi de $18,0 \%$. O teste do Qui-Quadrado, calculado a partir dos valores absolutos, ao comparar o número de crianças anêmicas, em cada um dos momentos do estudo, nas creches e UBS nảo demonstrou diferenças significantes.

Nas 39 crianças que receberam a suplementação, por um período de 9 meses, o percentual de anemia, 


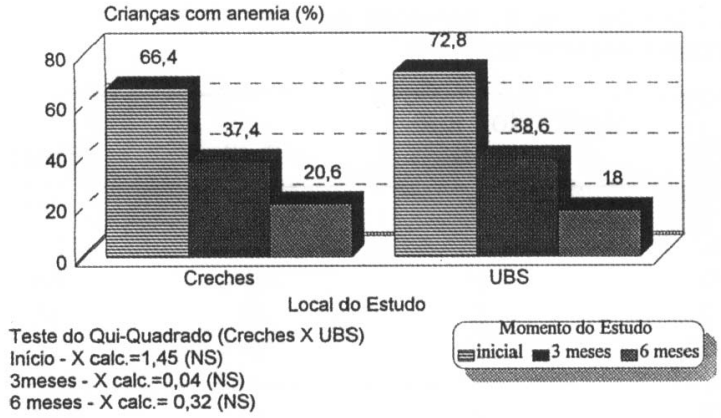

Flgura 1- Evoluçăo dos percentuais de crianças menores de 2 anos com anemia, nos momentos inicial e após 3 e 6 meses de uso do leite em pó fortificado, Grande São Paulo e Unidade Básica de Saúde (UBS) de Ibiúna, SP.

que, no momento anterior à intervençāo, erade 79,5\%, reduziu-se para $43,6 \%$ aos 3 meses, $33,3 \%$ aos 6 meses e 2,6\% aos 9 meses de uso do leite em pó fortificado com ferro e vitamina $\mathrm{C}$ (Figura 2). O teste de Cochram demonstrou que este decréscimo foi significante, com um $\mathrm{G}$ calculadode $46,68(\mathrm{P}<0,001)$.

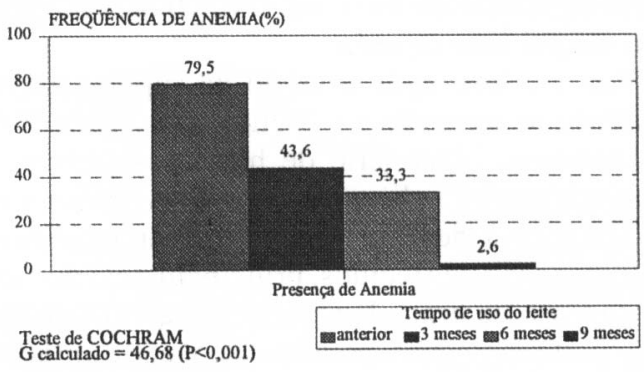

Figura 2 - Percentuais de crianças menores de 2 anos com anemia, no momento anterior à intervençăo $\theta$ após 3,6 e 9 meses de utilização do leite fortificado, na Unidade Básica de Saúde de Ibiúna, SP.
A Tabela 5 registra a evolução da condição nutricional das 107 crianças das creches, avaliada pelo critério de Gomez, onde pode-se verificar acréscimos importantes nas proporçōes de crianças com sobrepeso ( 7 no momento inicial e 23 ao final) e normais (de 43 para 73). Por outro lado, o total de desnutridos leves reduziu-se de 43 para 8 e o de moderados e graves de 14 para 3. O teste de Kappa mostrou concordância significante $(\mathrm{P}<0,001) \mathrm{com}$ um percentual de $57,0 \%$ de crianças melhoradas e apenas $1,9 \%$ de pioradas. O teste de McNemar demonstrou que o percentual de crianças que melhoraram foi significantemente maior do que as que pioraram ao final da intervençåo.

A Tabela 6 repete as informações da tabela anterior para o grupo da UBS. Ela indica que foram irrelevantes as alteraçőes na condição nutricional das crianças acompanhadas. $O$ teste de Kappa encontrou percentual de $11,4 \%$ de crianças que apresentaram melhora na sua condiçảo nutricional, $18,8 \%$ que pioraram e $70,6 \%$ de inalterados, com uma alta concordância estatística $(\mathrm{P}<0,001)$. Por outro lado, ao comparar o percentual de crianças que apresentaram melhora na sua condição nutricional com as que pioraram, o teste de McNemar năo revelou significância estatística.

\section{Comentários e Concluš̋es}

As prevalências de anemia encontradas no momento inicial do estudo foram superiores às constatadas, na mesma faixa etária, em UBS do Estado de Sāo Paulo ${ }^{24,25}$, detectada em 1993. Refletem, também, o aumento da prevalência constatado na região nos últimos 20 anos ${ }^{11,20}$.

A ocorrência de um percentual maior de crianças com hemoglobinas inferiores a $9,5 \mathrm{~g} / \mathrm{dl}$ nas creches, onde a média das idades foi de 13 meses e de hemoglobinas entre 9,5 e $10,9 \mathrm{~g} / \mathrm{dl}$ na UBS de

Tabela 5 - Distribuição das crianças acompanhadas nas creches da Grande São Paulo, segundo sua condiçăo nutricional (Gomez), nos momentos iniclal e final da intervençāo.

\begin{tabular}{ccccccc} 
Momento & \multicolumn{7}{c}{ Momento Final } \\
Inicial & Sobrepeso & Eutrófico & D-I & D-II & D-III & Total \\
\hline Sobrepeso & 5 & 2 & 0 & 0 & 0 & 7 \\
Eutrofico & 13 & 30 & 0 & 0 & 0 & 43 \\
D-1 & 5 & 32 & 6 & 0 & 0 & 43 \\
D-II & 0 & 9 & 2 & 3 & 0 & 14 \\
D-III & 0 & 0 & 0 & 0 & 0 & 0 \\
\hline Total & 23 & 73 & 8 & 3 & 0 & 107
\end{tabular}

D.-I, D.-II e D-III - Níveis de desnutriçăo: leve (I); moderado (II), grave (III)

Teste Kappa $-\mathrm{K} w=0.226 \cdot$ Zcalc $=11.93 \quad(\mathrm{P}<0.001)$

$\%$ de melhorados $=\mathbf{5 7 . 0 \%}$

$\%$ de piorados $=1.9 \%$

$\%$ de inalterados $=41.1 \%$

Teste de McNemar $-X^{2}$ calc. $=55.25 \quad(P<0.001)$ 
Tabela 6 - Distribuição das crianças acompanhadas na UBS de lbiúna, segundo sua condlçāo nutricional (Gomez), nos momentos inicial e final da intervençăo e testes estatísticos.

\begin{tabular}{ccccccr}
$\begin{array}{c}\text { Momento } \\
\text { Inicial }\end{array}$ & Sobrepeso & Eutrófico & D-I & Momento Final & D-III & Total \\
\hline Sobrepeso & 12 & 20 & 0 & 0 & 0 & 32 \\
Eutrófico & 5 & 98 & 20 & 1 & 0 & 124 \\
D-I & 0 & 13 & 45 & 0 & 0 & 58 \\
D-II & 0 & 1 & 6 & 3 & 0 & 10 \\
D-III & 0 & 0 & 1 & 3 & 0 & 4 \\
\hline Total & 17 & 132 & 72 & 7 & 0 & 228
\end{tabular}

D.-I, D.-II, D.-III - Níveis de desnutrição: leve (I); moderado (II); grave (III)

Teste Kappa $-\mathrm{KW}=0.58$ - Zcalc. $=10.44-(\mathrm{P}<0.001)$

$\%$ de melhorados $=11.4 \%$

$\%$ de piorados $=18.0 \%$

$\%$ de inalterados $=70.6 \%$

Teste de McNemar $-X^{2}=2.06$ (NS)

Ibiúna, onde a média das idades foi de 8,5 meses, indica que, além de estar instalando-se, mais precocemente, a anemia manifesta-se com gravidade mais elevada nas crianças maiores de um ano. Certamente estas respostas ocorrem pelo fato de crianças, nascerem com baixas reservas de ferro, estarem sendo amamentadas por um período médio de dois meses, bem como, receberem uma dieta substitutiva basicamente láctea, com ínfimas quantidades de ferro, até completarem dois anos ${ }^{18}$.

A estratégia de utilizar a distribuição mensal de algum tipo de suplemento alimentar, como forma de atrair a populaçāo mais carente do ponto de vista socioeconômico as UBS, e com isso melhorar a atençāo à saúde da criança, encontra forte respaldo no presente estudo. Seria praticamente impossivel, conseguir que 241 mães comparecessem mensalmente a uma UBS e ao constatar ao final de 6 meses, que apenas $13(5,4 \%)$ desistiram do suplemento.

O impacto do leite fortificado sobre a condição hematológica das crianças acompanhadas revela que pequenas quantidades de ferro/dia são capazes de recuperar e prevenir a anemia em menores de 2 anos $^{1}$. Uma ingestão suplementar diária entre 6 e 12 $\mathrm{mg} / \mathrm{Fe} / \mathrm{dia}$ ( 2 a 4 mamadeiras de $250 \mathrm{ml}$ ), por um período de 6 meses, foi suficiente para reduzir significantemente a carência de ferro na população estudada, tanto nas creches, como na UBS. Se o período de uso do leite fortificado for de 9 meses, consegue-se controlar esta deficiência na população usuária. Esta afirmação é baseada em em outro estudo ${ }^{25}$, realizado com 620 crianças com idades compreendidas entre 6 e 36 meses, em Unidades de Saúde do Município de São Paulo, demonstrando que apenas $3 \%$ das anemias na faixa etária estudada não eram ferropênicas.

O comportamento das médias das hemoglobinas nos momentos inicial $\mathrm{e}$ final com um acréscimo médio de $1,3 \mathrm{~g} / \mathrm{dl}$ nas creches e $1,1 \mathrm{~g} / \mathrm{dl}$, na UBS, num período de 6 meses, reforçam as afirmaçōes anteriores.
A análise comparativa do incremento da hemoglobina no grupo de crianças com anemia revelou aumento médio de $1,9 \mathrm{~g} / \mathrm{dl}$ nas creches e de $1,4 \mathrm{~g} /$ d1 na UBS, enquanto que para as crianças sem anemia este valor foi de $0,3 \mathrm{~g} / \mathrm{dl}$ nas creches e de $0,49 \mathrm{~g} / \mathrm{dl}$ na UBS, revelando que o grupo de crianças anêmicas apresenta um poder de absorção muito maior do ion ferro que a verificada no grupo de crianças sem anemia.

Nas creches, após o início da suplementação, observou-se aumento no apetite e atividade física das crianças. Uma série de hipóteses podem ser levantadas para explicar este fato. Provavelmente, a suplementação de ferro proporcionou uma melhoria na oxigenaçăo dos tecidos, principalmente do sistema nervoso central ${ }^{3}$. Estes fatores, aliados a dieta com aporte energético-protéico adequadoe um acompanhamento diário por profissionais especializados, forneceram condições para a reduçāo da freqüência da Desnutrição Energético-Protéica (DEP), registrada ao final da pesquisa. Os resultados indicam que a melhoria da condição hematológica trouxe, como conseqüência, a recuperação do estado nutricional, apontando a relaçåo existente entre estas duas patologias, ou seja, a carência de ferro sendo uma das responsáveis pelo aparecimento da DEP.

A melhora na condiçảo nutricional não se repetiu nas crianças acompanhadas na UBS de Ibiúna. Alguns fatores poderiam explicar este fato: o baixo poder aquisitivo e de educação nutricional da populaçāo, trazendo como consequência uma dieta deficiente e a alimentação basicamente láctea recebida pelas crianças até completarem 2 anos e a presença da diluiçăo intrafamiliar do leite recebido.

O leite em p 6 fortificado com ferro e vitamina $C$ mostrou-se excelente veículo na prevenção e controle da anemia carencial ferropriva, com muitas vantagens sobre as outras formas de intervenção. A suplementaçāo medicamentosa exige a colaboração da mãe e a obrigatoriedade de se administrar o 
fármaco por um período prolongado, o que em nossa experiência foram os principais fatores do insucesso deste tipo de intervenção ${ }^{25}$. Por outro lado, o uso do leite fortificado permite que o alimento seja o veículo para a prevenção da anemia, liberando a māe dessa responsabilidade e preocupaçāo. Além disso, a diferença de preço entre o leite em pó integral e o fortificado foi de cerca de $4,0 \%$, o que pode tornar-se irrisório frente aos benefícios obtidos.

Assim, o caminho da fortificação de alimentos é um dos mais promissores para o controle das carências nutricionais de oligo-elementos da população infantil do Brasil e, talvez, do Terceiro Mundo.

\section{Referênclas Blbllográflcas}

1. AMERICAN ACADEMY OF PEDLATRICS. Committe on Nutrition. Iron supplementation for infants. Pediatrics, 58: $765-8,1976$

2. BATISTA FILHO, M. \& BARBOSA, N. P. Pró-Memória: alimentação e nutrição no Brasil: 1974-1984. Brasília, INAN, 1985.

3. BRANDALISE, S.R. \& MATSUDA, E. Anemias carenciais In: Nobrega, F. J. Desnutriçdo intra-sterina e pos-natal. São Paulo, R.S. Distr. e Ed., 1985. p. 427-35.

4. COOK, J. D. \& REUSSER, M. E. Iron fortification: an update. Am. J. Clin. Nktr, 38: 648-59, 1983.

5. DeMAYER, E. M. Preventing and controling inon deficiency anaemia through primary care. Ginebra, OMS, 1989.

6. GOMEZ. F. Desnutrición. Bol. Méd. Hosp. Infant. Méx., 3: 543-51,1946.

7. HAILINE, A. Standard methods of clinical chemistry, New York, Academic Press, 1958.

8. JOĀO, W. S. J. Prevalência de anemia na populaçăo atendida nos postos de saúde da Secretarja de Saúde do Estado do Pará e em escolares participantes do Programa Nacional de Alimentaçăo Escolar; Relatório Anual. Convênio INAN/UFPA. Belem, 1983.

9. LANDIS, J, R. \& KOCK, G. G. The measurement of observer agreernent for categorical data. Biometrics, 33: 159-74, 1977.

10. MAcPHAIL, A. P.\& BOTHWELL, T. H. Fortification of the diet as a strategy for preventing iron deficiency. Acto Paediatr. Scand. Suppl, (361): 114-24,1989.
11. MONTEIRO, C. A. Saùde e nutriçäo das crianças de São Paulo: diagnóstico, contrastes sociais e tendências. Săo Paulo, L. Hucitec / Ed. USP, 1988.

12. NESTLE NUTRITION. El hierro en la alimentación del lactante y del niño. Sāo Paulo, 1984.

13. OLIVARES, $M$. et al. Prevention of iron deficiency by milk fortification. Acta Paediatr.Scand. Suppl, (361): 109$13,1989$.

14. ORGANTZACIÓN MUNDIAL DE LA SALUD. Lucha contra la anemia nutricional, especialmente contra la carencia de hierro. Ginebra, 1975. (OMS - Serie de Informes Tecnicos, 580).

15. ORGANIZACIÓN MUNDIAL DE LA SALUD, Medicion del efecto nutricional de programas de suplementacion alimentaria a grupos vuinerables. Ginebra, 1980.

16. REMINGTON, R, D. \& SCHORK, M. A. Statistics with applications to biological and health sciences. Englewood Cliffs, Prentice-Hall Inc, 1970.

17. ROMANI, S. A. M. et al. Anemias em pre-escolares: diagnóstico, tratamento e avaliação. Recife - Pe, Brasil. Arch. Latinoam. Nutr., 67: 159-67, 1991.

18. SECRETARLA DE ESTADO DA SAÚDE. Centrode Apoio ao Desenvolvimento da Atenção Primária à Sacide. Grupo de Atençāo à Nutriçāo. Sistema de vigilâncía alimentar e nutricional. Såo Paulo, 1993. [dados inéditos].

19. SIEGEL, S. Estadistica no paranemica. Mexico, Ed. Trillas, 1975.

20. SIGLLEM, D. M. et al. Anemia ferropriva en crianças no Município de São Paulo. Rev. Saúde Pública, 12: 16878, 1978 .

21. SOKAL, R. R., \& ROHLF, F. J. Biometry. San Francisco, W. H. Freeman and Company, 1969.

22. STEKEL, A. et al. Prevention of iron deficiency by milk fortification. II A field trial with a full- fat acidified milk. Am. J. Clin. Nutr., 47: 265-9, 1988.

23.TORRES, M. A. A. Estado nutricional e aspectos socioeconómicos de famflias rurais do Trópico Semi-Árido (Nordeste do Brasil). Recife,1982. [Dissertação de Mestrado - Universidade Federal de Pernambuco].

24. TORRES, M. A. A., et al. Anemia em crianças menotes de 2 anos atendidas nas unidades básicas de saúde no Estado de Sāo Paulo. Rev. Saúde Pública, 28: 290-4,1994.

25. TORRES, M. A. A., et al. Terapęutica com doses profilaticas de sulfato ferroso, como medida de intervençäo no combate à carência de ferro, em crianças atendidas em unidades básicas de saúde. Rev. Saúde Pública, 28: 410$5,1994$.

26. TURCONI, S. J. \& TURCONI, V. L.Anemia ferropriva: incidência em uma populaçāo infantil. Pediatr. Mod., 28: 107-12,1992.

\section{Abstract}

The impact of the use of fortfied powdered whole milk ( $9 \mathrm{mg}$ of iron and $65 \mathrm{mg}$ of vitamin $\mathrm{C} / 100 \mathrm{~g}$ of milk) on the hemogiobin levels of children under 2 years of age was evaluated, over a period of 6 months, in 107 chlldren enrolled in municipal Day Care Centers (DCC) and in 228 seen at a Basic Health Care Unit (BHCU). Before the beginning of the intervention, 66.4\% of the children in the DCC and $72.8 \%$ of those seen at the BHCU had hemoglobin levels under $11.0 \mathrm{~g} / \mathrm{dl}$. After 6 months of fortified milk intake, these percentages fell to $20.6 \%$ and $18.0 \%$ respectively. The average hemoglobin before the intervention was 10.3 $\mathrm{g} / \mathrm{d} / \mathrm{in}$ the DCC and 10.5 in the BHCU. After 6 months these increased to $11.6 \mathrm{~g} / \mathrm{d}$ in the populations studied. Conceming the nutritional condition, evaluated according to Gomez's criteria, $57 \%$ of the DCC children presented an improvement, $41.1 \%$ showed changens and only $1.9 \%$ became worse. In the $B H C U, 11.4 \%$ presented better condition, $70.6 \%$ remained the same and $18 \%$ worsened, which demonstrated differences in response regarding improvememt of nutritional condition, when fortified milk was used in closed and open environments. The authors conclude that the utilization of enriched foods is an exceflent altemative in the treatment of iron deficiency in populations of children under 2 years of age. 